\title{
ASPECTOS Y NORMAS DE ACCESIBILIDAD WEB
}

\author{
Jairo Armando Riaño Herrera \\ Universidad Pedagógica y Tecnológica de Colombia \\ jairo.riano@uptc.edu.co
}

\author{
Javier Antonio Ballesteros Ricaurte \\ Universidad Pedagógica y Tecnológica de Colombia \\ javier.ballesteros@uptc.edu.co
}

(Tipo de Artículo: Revisión. Recibido el 04/11/2014. Aprobado el 01/12/2014)

\begin{abstract}
RESUMEN
Este artículo revisa la diferencia y relación que existe entre los términos de accesibilidad y usabilidad en contextos de software y páginas web. Además con base en la definición de accesibilidad web hace una revisión de las normas y estándares que existen y que son mantenidas por el consorcio internacional W3C con su iniciativa "Web Accessibility Inititive", con el objetivo de garantizar la accesibilidad a los sitios web por cualquier persona usuario independiente de su estado de discapacidad. Finalmente se enumeran aspectos a tener en cuenta al momento de publicar contenidos en la web y algunas herramientas de validación en línea que permiten validar el cumplimiento de normas de accesibilidad de los sitios web.
\end{abstract}

Palabras clave. Sitios web, accesibilidad, usabilidad, estándares.

\section{WEB ACCESSIBILITY ASPECTS AND REGULATIONS}

\begin{abstract}
This article reviews the difference and relations between the terms of accessibility and usability in the context of software and web pages. Furthermore, based on the definition of web accessibility is performed a review of the existing rules and standards and that are maintained by the international consortium W3C with its "Web Accessibility Initiative" in order to ensure the accessibility of websites by any user independently of his disability status. Finally are listed some aspects to consider when publishing contents on the web and some online validation tools for validating compliance with website accessibility standards.
\end{abstract}

Keywords. Websites, Accessibility, Usability, Standards.

\section{Aspects et règles d'accessibilité du Web}

\begin{abstract}
Résumé
Cet article révise la différence et relation qui existe entre les termes d'accessibilité et utilisabilité dans contextes de software et pages web. Par ailleurs d'après la définition d'accessibilité web on fait une révision des règles et standards existants et qui sont maintenus par le consortium international W3C ave son initiative "Web Accesibility Initiative ", dans l'intention de garantir l'accessibilité aux pages web quels qu'en soient les utilisateurs indépendamment de leur type de handicap. Après on présente des aspects à considérer pour publier des contenus sur Internet et quelques outils de validation en ligne qui permettent de valider l'accomplissement des règles d'accessibilité des pages web.
\end{abstract}

Mots-clés. Pages web, accessibilité, utilisabilité, standards. 


\section{INTRODUCCIÓN}

El avance en las tecnologías de la información y las comunicaciones permiten acceder en forma rápida a contenidos informativos publicados en la web y hacer uso de herramientas de comunicación entre personas en cualquier parte del mundo. Para cierto porcentaje de usuarios el acceso a éste mundo de posibilidades de información es restringido por tener algún tipo de discapacidad física, sensorial, cognitiva o tecnológica, lo que les impide usar en forma adecuada las tecnologías y cumplir con su objetivo de usabilidad.

Sin embargo, existen normas y estándares de accesibilidad web que son mantenidas por la organización internacional W3C (World Wide Web Consortium) con el objetivo de ofrecer pautas a seguir por parte de los desarrolladores web para que los sitios web sean accesibles a cualquier usuario que desee consultar sus contenidos incluso cuando tenga algún estado de discapacidad.

\section{USABILIDAD Y ACCESIBILIDAD}

Los términos de usabilidad y accesibilidad se encuentran relacionados de una manera que en ocasiones se puede llegar a confundir el significado de lo que se desea expresar.

Para la usabilidad, la Organización Internacional para la Estandarización (ISO), ofrece dos definiciones: en la norma ISO/IEC 9241 [1] "Usabilidad es la eficacia, eficiencia y satisfacción con la que un producto permite alcanzar objetivos específicos a usuarios específicos en un contexto de uso específico". La otra definición se encuentra en la norma ISO/IEC 9126 [2] "La usabilidad se refiere a la capacidad de un software de ser comprendido, aprendido, usado y ser atractivo para el usuario, en condiciones específicas de uso". Con base en las dos definiciones anteriores, se destaca la importancia de la usabilidad en el software, porque permite a los usuarios lograr cumplir con los objetivos de uso en un programa específico.

La accesibilidad por su parte, aplica para contenidos de páginas web, los cuales deben ser accesibles para los usuarios independientemente de sus capacidades físicas, sensoriales o tecnológicas. La accesibilidad es de gran relevancia porque muchos de los sistemas de información interactúan con los usuarios intercambiando información a través de páginas web, que de no ser éstas accesibles, no permitirían cumplir con los objetivos específicos reduciendo de ésta manera la usabilidad del producto de software.

La relación que existe entre usabilidad y accesibilidad permite que un aumento en el grado de accesibilidad, aumente también el nivel de usabilidad de un sistema.

\section{ACCESIBILIDAD WEB}

La accesibilidad web permite que la información, recursos y/o servicios se pueden encontrar en los sitios web a nivel de Internet o de una Intranet. Esta característica permite un acceso universal a la web independientemente del tipo de hardware, software, infraestructura de red, idioma, cultura, localización geográfica y capacidades de los usuarios [3]. La accesibilidad web permite que una página web sea utilizada para cualquier persona con condiciones que dificulten acceder a la información.

Existen problemas de accesibilidad a los contenidos de la web cuando por ejemplo:

- Las imágenes no tienen texto que describan su contenido, cuando quien está accediendo a la información es una persona en estado de discapacidad visual, que utiliza herramientas tecnológicas como un lector de pantalla para acceder a los contenidos web.

- La persona que navega tiene imposibilidad para usar un teclado o un ratón.

- Se utilizan expresiones que indican acciones sobre algún tipo de color específico, por ejemplo, "pulse sobre el botón de color azul" y la persona está accediendo a través de un dispositivo de pantalla monocromática.

- El lenguaje utilizado para describir los contenidos de la página es ambiguo, dificultando a personas con baja comprensión de lectura.

- Se usan elementos html en forma inadecuada, por ejemplo, tablas para el diseño u organización de los contenidos de las páginas web, ya que una herramienta tecnológica como el lector de pantalla no interpretaría adecuadamente los contenidos de la tabla.

\subsection{Web Accessibility Initiative (WAI)}

Con el objetivo de mantener y promover la accesibilidad web, la organización internacional World Wide Web Consortium (W3C), ha impulsado la Web Accessibility Initiative (WAI) [4] o Iniciativa para la Accesibilidad Web, encargada de proporcionar pautas y recursos que ayudan a hacer la web accesible. A través de foros internacionales promueve la colaboración entre la industria, organizaciones de personas en estado de discapacidad, investigadores de accesibilidad, organizaciones del gobierno y personas interesadas en los temas de accesibilidad web.

\subsection{Web Content Accessibility Guidelines (WCAG)}

Las Pautas de Accesibilidad al Contenido en la Web, son el conjunto de normas y pautas promovidas por el consorcio W3C para promover y difundir las directrices en el diseño de sitios web accesibles. A la fecha se han publicado dos versiones, la WCAG 1.0 y WCAG 2.0. La última versión es considerada un referente definitivo en el cual se deben basar los diseñadores web y personas encargadas de publicar información en la web. 


\subsubsection{Web Content Accessibility Guidelines $\mathbf{1 . 0}$}

La última versión del documento WCAG 1.0 fue publicada el 5 de mayo de 1999 [5] y se organiza en una serie puntos de verificación, prioridades, niveles y pautas.

Puntos de verificación. Indican los aspectos a tener en cuenta para cumplir con la pauta respectiva, estos aspectos se deben tener en cuenta en el momento de evaluar la accesibilidad de una página web en forma manual o automática.

Prioridades. Cada punto de verificación tiene un nivel de prioridad que indica la importancia de contemplar el punto de verificación en la accesibilidad de los contenidos web, siendo los de nivel 1 los de mayor importancia. Los niveles de prioridad son tres:

- Prioridad 1. Los desarrolladores de contenidos web tienen que satisfacer este punto de verificación, ya que de otra manera, uno o más grupos de usuarios encontrarán imposible acceder a la información o contenido web.

- Prioridad 2. Los desarrolladores de contenidos web tienen que satisfacer este punto de verificación, ya que de no hacerse uno o más grupos encontrarán dificultades en el acceso a la información del documento.

- Prioridad 3. Los desarrolladores de contenidos web tienen que satisfacer este punto de verificación, ya que de no hacerse uno o más grupos de usuarios encontrarán alguna dificultad para acceder a la información del documento.

Con base en lo anterior, un sitio web que no cumple con al menos la prioridad 1, no es accesible para un gran número de usuarios con ciertos niveles de discapacidad, por ejemplo, para una persona que tiene una discapacidad visual le sería imposible acceder a información a través de colores, o no podría percibir lo que el autor pretende dar a conocer a través de una imagen si ésta no cuenta con una descripción alternativa.

Un sitio web que cumple como mínimo la prioridad 1 permite el acceso a los contenidos, aunque ciertos usuarios encuentren alguna dificultad para el acceso.

El acceso a los contenidos web se puede comparar como el acceso a una edificación que solamente cuenta con escaleras para ir a los pisos superiores, un usuario en silla de ruedas encontraría imposible acceder por sus propios medios. Pero, si además de escaleras provee una rampa, la misma persona tendría la posibilidad de acceso por sus propios medios, aunque requiere de esfuerzo físico, en éste caso, la rampa se asocia con un primer nivel de prioridad. Ahora, si además la rampa es una banda eléctrica, aumentará el nivel de accesibilidad y por lo tanto el nivel de prioridad. Por último, un ascensor permitiría el acceso con total facilidad y estaría asociado al tercer nivel de prioridad ya que la persona en silla de ruedas encontraría facilidad para acceder a la edificación.

Niveles de Adecuación. Los niveles de adecuación permiten establecer el cumplimiento de los puntos de verificación asociados a una prioridad. Existen tres niveles de adecuación:

- Nivel A. Se satisfacen todos los puntos de verificación de prioridad 1

- Nivel AA. Se satisfacen todos los puntos de verificación de prioridad 1 y 2

- Nivel AAA. Se satisfacen todos los puntos de verificación de prioridad 1, 2 y 3 .

La W3C recomienda que las páginas informen en forma adecuada el cumplimiento de los puntos de verificación, y para hacerlo se puede de una de dos formas [5]:

Forma 1. Se debe especificar:

- El título de las pautas: "Web Content Accessibility Guidelines 1.0"

- La URL de las pautas: http://www.w3.org/TR/1999/WAI-

WEBCONTENT-19990505

- El nivel de adecuación que se satisface "A", "AA" o "AAA"

- El alcance cubierto por la información (página, sitio o porción definida del sitio)

Forma 2: Incluir en cada página que afirme su conformidad uno de los tres logos proporcionados por la W3C indicando el nivel de cumplimiento. Los logos se pueden descargar desde el sitio respectivo [6] dispuesto por la W3C.

Las siguientes son las Pautas de Accesibilidad al Contenido web en la versión 1.0:

1. Proporcione alternativas equivalentes para el contenido visual y auditivo

2. No se base sólo en el color

3. Utilice marcadores y hojas de estilo y hágalo apropiadamente

4. Identifique el idioma usado

5. Diseñe tablas que se transformen correctamente

6. Asegúrese de que las páginas que incorporan nuevas tecnologías se transformen correctamente

7. Asegure al usuario el control sobre los cambios de los contenidos tempo-dependientes

8. Asegure la accesibilidad directa de las interfaces de usuario incrustadas

9. Diseñe para la independencia del dispositivo

10. Utilice soluciones provisionales

11. Utilice las tecnologías y pautas $W 3 C$

12. Proporcione información de contexto y orientación

13. Proporcione mecanismos claros de navegación

14. Asegúrese de que los documentos sean claros y simples 
Resumiendo, cada pauta tiene unos puntos de verificación para establecer si el contenido es accesible con base en la pauta, el punto de verificación se asocia a un nivel de prioridad, para de ésta manera determinar el nivel de adecuación correspondiente (A, AA, AAA). Las verificaciones se pueden realizar en forma manual o automática a través de herramientas o validadores de accesibilidad como HERA [7], o el servicio de validación en línea proporcionado por la misma W3C [8].

\subsubsection{Web Content Accessibility Guidelines 2.0}

La WCAG 2.0 es la última versión de las pautas de accesibilidad publicada por la W3C el 11 de diciembre de 2008 [9]. Existe también la traducción candidata a ser la oficial en español publicada el 15 de diciembre de 2009 [10].

Según la información publicada en el sitio de la Universidad de Alicante [11] el 15 de octubre de 2012 las pautas de accesibilidad al contenido web en su versión 2.0, habían sido aprobadas como un estándar internacional en la ISO/IEC 40500:2012.

La versión 2.0 de las pautas de accesibilidad se basan en la versión 1.0 y fueron diseñadas con el objetivo de ser aplicadas en una amplia gama de tecnologías web de ahora y del futuro.

La WCAG 2.0 está compuesta por varios niveles de orientación: principios generales, pautas generales, criterios de conformidad y técnicas suficientes y recomendables. Los principios generales son el mayor nivel en las pautas de accesibilidad y están conformados por directrices y criterios de cumplimiento. Los principios generales son cuatro:

- Perceptibilidad. Indica que los contenidos y componentes de la interfaz de usuario deben presentarse de manera que sean percibidos, se componen de cuatro directrices y 22 criterios de cumplimiento.

- Operabilidad. Indica que los componentes de la interfaz de usuario y la navegación deben ser operables, se componen de cuatro directrices y 20 criterios de cumplimiento.

- Comprensibilidad. Indica que la información y el manejo de la interfaz de usuario deben ser comprensibles, se componen de tres directrices y 17 criterios de cumplimiento

- Robustez. Indica que el contenido debe ser lo suficientemente robusto para confiarse en su interpretación por parte de una amplia variedad de agentes de usuario como navegadores, se componen de una directriz y dos criterios de cumplimiento.

En el siguiente nivel de los principios generales se encuentran las doce pautas, proporcionando objetivos básicos que los diseñadores de los sitios deben lograr para crear contenidos más accesibles para los usuarios con distinta discapacidad. Aunque estas pautas no son verificables proporcionan un marco y objetivos generales como ayuda para comprender los criterios de conformidad.

Los criterios o requisitos de conformidad se proporcionan para cada pauta, son verificables y se pueden emplear en situaciones en las que existen requisitos y necesidad de evaluación de conformidad, por ejemplo: especificaciones de diseño, compras, regulación o acuerdos contractuales. Se definen tres niveles de conformidad: el más bajo es el " $A$ ", siguiendo el "AA" y "AAA", cada nivel o criterio de conformidad se debe cumplir en forma completa:

- Nivel A. La página web satisface todos los criterios de conformidad del nivel A o proporciona una versión alternativa conforme.

- Nivel AA. La página web satisface todos los criterios de conformidad de los niveles A y AA o proporciona una versión alternativa conforme.

- Nivel AAA. La página web satisface todos los criterios de conformidad de los niveles A, AA y AAA o proporciona una versión alternativa conforme.

En cuanto a las técnicas suficientes y recomendables son informativas y se agrupan en dos categorías: las que son suficientes para satisfacer los criterios de conformidad y las que son recomendables que permiten a los autores afrontar mejor las pautas.

Según la WCAG 2.0 [9], para que una página web cumpla con las directrices de accesibilidad WCAG 2.0 debe cumplir con los siguientes requisitos de conformidad [12].

1. Cumplir o satisfacer la totalidad de alguno de los niveles de conformidad A, AA o AAA.

2. Los niveles de conformidad se aplican a las páginas completas y no pueden ser alcanzados si se excluye una parte de la página.

3. Cuando varias páginas web conforman un proceso que se debe seguir, todas las páginas deben cumplir con el nivel especificado o uno superior.

4. Solo se puede depender de las tecnologías usadas de forma compatible con la accesibilidad para satisfacer los criterios de conformidad.

5. Si las tecnologías se usan de una forma que no es compatible con la accesibilidad, o están usadas de una forma que no cumple los requisitos de conformidad, no deben impedir a los usuarios acceder al contenido del resto de la página.

A continuación se encuentra una descripción de las pautas de accesibilidad organizada jerárquicamente por principios y pautas:

Principio 1. Perceptible - La información y los componentes de la interfaz de usuario deben ser presentados a los usuarios de modo que ellos puedan percibirlos. 
- Pauta 1. Alternativas textuales - Se deben proporcionar alternativas textuales para todo contenido no textual de modo que se puedan convertir a otros formatos que las personas necesiten.

- Pauta 2. Medio tempo-dependiente - Se debe proporcionar alternativas para los medios tempodependientes.

- Pauta 3. Adaptable - El contenido se debe crear de manera que se pueda presentar de diferentes formas (por ejemplo, con una disposición más simple) sin perder información o estructura.

- Pauta 4. Distinguible - Se debe facilitar a los usuarios ver y oír el contenido, incluyendo la separación entre el primer plano y el fondo.

Principio 2. Operable - Las pautas y criterios de conformidad de éste principio, se orientan a que los componentes de la interfaz de usuario y la navegación deben de ser operables.

- Pauta 1. Accesible por Teclado - Para toda funcionalidad del contenido o la interfaz de usuario se debe proporcionar acceso mediante el teclado.

- Pauta 2. Tiempo Suficiente - Se debe proporcionar a los usuarios el tiempo suficiente para leer y usar el contenido.

- Pauta 3. Convulsiones - No se debe diseñar contenido de un modo que se sepa, podría provocar ataques, espasmos o convulsiones.

- Pauta 4. Navegable - Se deben proporcionar medios para ayudar a los usuarios a navegar, encontrar contenido y determinar donde se encuentran

Principio 3. Comprensible - Las pautas y criterios de conformidad de éste principio, se orientan a que la información y el manejo de la interfaz de usuario deben ser comprensibles.

- Pauta 1. Legible - Se debe hacer que los contenidos textuales resulten legibles y comprensibles.

- Pauta 2. Predecible - Se debe hacer que las páginas web aparezcan y operen de manera predecible.

- Pauta 3. Entrada de datos asistida - Ayudar a los usuarios a evitar y corregir errores.

Principio 4. Robusto - Las pautas y criterios de conformidad de éste principio, se orientan a que el contenido debe ser suficientemente robusto como para ser interpretado de forma fiable por una amplia variedad de aplicaciones de usuario, incluyendo las ayudas técnicas.

- Pauta 1. Compatible - Se debe maximizar la compatibilidad con las aplicaciones de usuario actuales y futuras, incluyendo las ayudas técnicas.
Es necesario informar el nivel de accesibilidad que cumple el sitio web. Tal como se había descrito anteriormente, se puede hacer de alguna de dos formas, de las cuales para la segunda se utilizan logos o imágenes estandarizadas por la W3C las cuales se describen en la tabla 1.

\section{Tabla 1. Logos de conformidad con directrices de accesibilidad. Fuente [13].}

\begin{tabular}{|c|c|c|}
\hline $\begin{array}{c}\text { Nivel de } \\
\text { Conformidad A }\end{array}$ & $\begin{array}{c}\text { Nivel de } \\
\text { Conformidad AA }\end{array}$ & $\begin{array}{c}\text { Nivel de } \\
\text { Conformidad AAA }\end{array}$ \\
\hline W3C $\begin{array}{c}\text { WAI-A } \\
\text { WCAG 2.0 }\end{array}$ & W3C $\begin{array}{c}\text { WAI-AA } \\
\text { WCAG 2.0 }\end{array}$ & W3C WAI-AAA \\
\hline
\end{tabular}

El uso de las imágenes especificadas en la tabla anterior se debe realizar con absoluta responsabilidad por parte de las personas $u$ organizaciones encargadas de publicar contenidos en la web, ya que nada impide que se puedan insertar en una página web sin que ésta cumpla con las normas que se especifican en el logo, sin embargo, a través de herramientas de terceros, de las cuales gran cantidad son avaladas por la W3C [14], se puede verificar el cumplimiento de los niveles de accesibilidad web y desde luego que no sería bien visto por la comunidad de usuarios un sitio que no cumple con lo que indica a través de un logo, esto indicaría una falta de seriedad y baja confiabilidad de la información publicada en el sitio web.

La accesibilidad web compete a tres instancias: quienes crean los navegadores o agentes de usuario, quienes crean las herramientas tecnológicas de apoyo para las personas con discapacidad y quienes generan los contenidos en las páginas web, cada instancia debe ser responsable de aplicar y tener en cuenta las normas de accesibilidad web promulgados por la W3C en sus versiones de WCAG 1.0 y 2.0.

La versión WCAG 2.0 publicada en diciembre de 2008 reemplazó a la 1.0 que se lanzó en mayo de 1999 y debería ser la versión a tener en cuenta por cada una de las instancias involucradas en aspectos de publicación de contenidos y accesibilidad web, teniendo en cuenta que en su última versión de pautas de accesibilidad, éstas están orientadas al uso de nuevas herramientas de acceso a los contenidos de las páginas web tal como los dispositivos móviles.

\section{HERRAMIENTAS DE VALIDACIÓN}

La organización internacional $\mathrm{W} 3 \mathrm{C}$ es la responsable del desarrollo y evolución de lo relacionado con las tecnologías de Internet y es la encargada de velar por el cumplimiento de las normas que la componen. Para lo anterior dispone de herramientas en línea para validar que las páginas web cumplan con los estándares de HTML en sus diferentes versiones, Hojas de Estilo de Cascada (CSS - Cascade Style Sheet) y Javascript. 
Para el caso del cumplimiento de las normas y pautas de accesibilidad web se hace uso de herramientas de terceros que se basan ya sea en WCAG 1.0 o WCAG 2.0. A continuación se describen algunas de las herramientas que se pueden usar para validación del cumplimiento de estándares de accesibilidad web:

- A-Checker - Es una herramienta de la Universidad de Toronto lanzada en el 2006 [15]. Evalúa el contenido HTML para encontrar problemas de accesibilidad. Se puede entrar la URL de la página, cargar un documento HTML o pegar el código de la página. Produce un informe de todos los problemas de accesibilidad encontrados basados en los criterios seleccionados, admite WCAG 1.0 y WCAG 2.0.

- EvalAccess 2.0 [16] - Es una herramienta en línea que permite una evaluación automática de la accesibilidad web de las páginas usando los estándares de la W3C en su versión WCAG 1.0. Permite evaluar una página o sitio web a partir de una URL, también valido código HTML que se puede pegar en la página de la herramienta.

- Hera [17]. - Es una herramienta que se puede trabajar como un plugin del navegador web y permite revisar el cumplimiento de las pautas de accesibilidad web conforme a las directrices de WCAG 1.0 y WCAG 2.0. Hera facilita la revisión manual proporcionando información acerca de los elementos a verificar, instrucciones sobre cómo realizar ese control y dos vistas modificadas de la página (una en modo gráfico, otra del código HTML) con los elementos más importantes destacados con iconos y colores distintivos.

- Examinator [18]. Herramienta de evaluación de accesibilidad de acuerdo a las WCAG 2.0. La herramienta es gratuita y está en español. La validación se realiza incluyendo la URL de la página a analizar.

- Total Validator [19]. Es una herramienta de validación de varios estándares entre ellos WCAG 1.0 y 2.0. Es una aplicación desarrollada en Java y se ejecuta sobre diferentes sistemas operativos como una aplicación stand-alone.

\section{CONCLUSIONES}

La accesibilidad a los contenidos de los sitios web se debe garantizar para cualquier usuario, de manera que pueda acceder a la información de los sitios web independiente de su estado de discapacidad.

La W3C mantiene las pautas y normas del desarrollo tecnológico de la red Internet, y establece directrices de accesibilidad que deben de cumplir los sitios web para que sean accesibles.

La accesibilidad web involucra no solo a personas con estados de discapacidad, sino también a quienes son incapaces de acceder a los contenidos web por carencia de los medios tecnológicos adecuados.
El cumplimiento de aspectos mínimos de accesibilidad asegura que toda persona independiente de su estado de discapacidad pueda acceder a los contenidos web.

Los sitios web deberían cumplir al menos con el nivel de conformidad $\mathrm{A}$, de manera que asegure el acceso a sus contenidos.

\section{REFERENCIAS}

[1] "ISO 9241-210:2010," ISO 9241-210:2010. [Online]. Available: http://www.iso.org/iso/home/store/catalogue_tc/cat alogue_detail.htm?csnumber $=52075$. [Accessed: 04-Aug-2014].

[2] "ISO/IEC TR 9126-4:2004." [Online]. Available: http://www.iso.org/iso/catalogue_detail.htm?csnum ber=3975. [Accessed: 20-Aug-2014].

[3] "Guía Breve de Accesibilidad Web," Guía Breve de Accesibilidad Web. [Online]. Available: http://w3c.es/Divulgacion/GuiasBreves/Accesibilida d. [Accessed: 20-Aug-2014].

[4] "Web Accessibility Initiative," Web Accessibility Initiative WAI - home page. [Online]. Available: http://www.w3.org/WAl/. [Accessed: 21-Aug-2014].

[5] "Web Content Accessibility Guidelines 1.0," Web Content Accessibility Guidelines 1.0. [Online]. Available: http://www.w3.org/TR/1999/WAlWEBCONTENT-19990505/. [Accessed: 21-Aug2014].

[6] "Logos de conformidad con las Directrices de Accesibilidad para el Contenido Web 1.0 del W3C," Logos de conformidad con las Directrices de Accesibilidad para el Contenido Web 1.0 del W3C. [Online]. Available: http://www.w3.org/WAI/WCAG1-Conformance.

[Accessed: 07-Sep-2014].

[7] "Revisando la accesibilidad con estilo," Revisando la accesibilidad con estilo. [Online]. Available: http://www.sidar.org/hera/. [Accessed: 07-Sep2014].

[8] "Feed Validation Service," W3C Feed Validation Service, for Atom and RSS. [Online]. Available: http://validator.w3.org/feed/. [Accessed: 07-Sep2007].

[9] "Web Content Accessibility Guidelines (WCAG) 2.0," Web Content Accessibility Guidelines (WCAG) 2.0. [Online]. Available: http://www.w3.org/TR/WCAG20/. [Accessed: 21Aug-2014].

[10] "Pautas de Accesibiidad para el contenido web (WCAG) 2.0," Pautas de Accesibiidad para el contenido web (WCAG) 2.0. [Online]. Available: http://www.sidar.org/traducciones/wcag20/es/.

[11] "Pautas de accesibilidad del contenido en la web 2.0," Accesibilidad web: WCAG 2.0. [Online]. Available:

http://accesibilidadweb.dlsi.ua.es/?menu=wcag2.0. [Accessed: 07-Sep-2014].

[12] "Niveles de Adecuación de WCAG 2.0," Accesibilidad web: Niveles de Adecuación de $\begin{array}{lll}\text { WCAG 2.0. [Online]. Available: } & \end{array}$ 
http://accesibilidadweb.dlsi.ua.es/?menu=niveles2.0. [Accessed: 08-Sep-2014].

[13] "W3C Web Content Accessibility Guidelines 2.0 Conformance Logos," W3C Web Content Accessibility Guidelines 2.0 Conformance Logos. [Online]. Available: http://www.w3.org/WAI/WCAG2-Conformance. [Accessed: 10-Sep-2014].

[14] "Complete List of Web Accessibility Evaluation Tools," Complete List of Web Accessibility Evaluation Tools. [Online]. Available: http://www.w3.org/WAl/ER/tools/complete. [Accessed: 10-Sep-2014].

[15] "Web Accessibility Checker," IDI Web Accessibility Checker: Web Accessibility Checker. [Online].
Available: http://achecker.ca/checker/index.php. [Accessed: 12-Sep-2014].

[16] "Eval Access 2.0," EvalAcces Home. [Online]. Available:

http://sipt07.si.ehu.es/evalaccess2/index.html. [Accessed: 12-Sep-2014].

[17] "Revisando la Accesibilidad con Estilo," Revisando la Accesibilidad con Estilo. [Online]. Available: http://www.sidar.org/hera/. [Accessed: 12-Sep2014].

[18] "Evaluación de la Accesibilidad Web," Examinator. [Online]. Available: http://examinator.ws/. [Accessed: 12-Sep-2014].

[19] "Total Validator," Total Validator. [Online]. Available: $\quad$ https://www.totalvalidator.com//. [Accessed: 12-Sep-2014]. 